\title{
TEMPERATURE DEPENDENCE OF MAGNETISATION REVERSAL AND GMR IN SPIN VALVE STRUCTURES
}

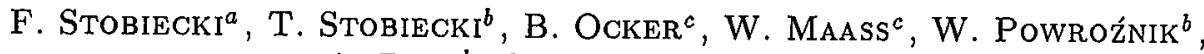 \\ A. PAJA ${ }^{d}$, C. $\mathrm{LOCH}^{e}$ AND K. RöLL ${ }^{e}$ \\ ${ }^{a}$ Institute of Molecular Physics, Polish Academy of Sciences \\ Smoluchowskiego 17, 60-179 Poznań, Poland \\ ${ }^{b}$ Department of Electronics, University of Mining and Metallurgy, Kraków, Poland \\ ${ }^{c}$ Balzers Process Systems GmbH, Alzenau, Germany \\ ${ }^{d}$ Department of Solid State Physics, Faculty of Physics and Nuclear Techniques \\ University of Mining and Metallurgy, Kraków, Poland \\ eDepartment of Physics, University of Kassel, Germany
}

\begin{abstract}
Temperature measurements of magnetisation reversal, resistance and magnetoresistance of $\mathrm{Co} / \mathrm{Cu} / \mathrm{Co} / \mathrm{FeMn}$ spin valve structures deposited in different conditions were performed. The influence of exchange anisotropy energy, interlayer coupling energy, and electron transport properties were taken into consideration in explaining differences in giant magnetoresistance effect of investigated samples.
\end{abstract}

PACS numbers: 75.70.Pa, 68.55.Ln, 68.55.-a

\section{Introduction}

Spin valve structures (SV) consisting of ferromagnetic/spacer/ferromagnetic/ antiferromagnetic layers are extensively studied due to their high amplitude and field sensitivity of giant magnetoresistance (GMR) effect. One of the crucial parameters in such SV is the ratio of exchange interlayer coupling energy $J$ to the unidirectional exchange anisotropy energy $E_{E B}, j=J / E_{E B}$. As it was demonstrated by Rijks et al. [1] both, the giant magnetoresistance amplitude $G M R_{A}=$ $100\left(R_{\max }-R_{\min }\right) / R_{\min }$ as well as the shape of $R(H)$ ( $R$ - resistance, $H$ magnetic field) are strongly dependent on the $j$ value. The interlayer coupling in SV was investigated by several groups (see e.g. [1, 2]). It was demonstrated that the origin of the dominating coupling (RKKY-like, magnetostatic and that caused by pinholes) depends strongly on thickness and structure of the spacer layer. Independently of the interlayer coupling GMR amplitude is also determined by'transport properties such as resistivity and mean free path of electrons. In this paper the magnetic and electrical transport properties of the $\mathrm{Co} / \mathrm{Cu} / \mathrm{Co} / \mathrm{FeMn} \mathrm{SV}$ deposited in different sputtering conditions are discussed. The origin of the differences in GMR effect for individual SV is explained on the base of the temperature dependencies of $J(T), E_{E B}(T), R(T)$ and supplementary measurements. 


\section{Experimental results and discussion}

The SV consisting of $\mathrm{Ta}(1) / \mathrm{Co}(1) / \mathrm{Cu}(1) / \mathrm{Co}(2) / \mathrm{FeMn} / \mathrm{Ta}(2) / \mathrm{Cu}(2)$ were deposited by sputtering in different conditions (Table). From a set of SV the four samples with essential differences in $G M R_{A}$ and/or deposition conditions were selected. The sputtering mode and thicknesses (determined by sputtering rate and confirmed by the small angle X-ray diffraction (SAXRD) measurements) of individual layers in each SV are collected in Table. The $m(H)$ ( $m$ - magnetic moment) and $R(H)$ dependencies were investigated for $H$ applied parallel to the exchange anisotropy axis with the use of the vibrating sample magnetometer and the d.c. four probe method. The magnetic and resistance measurements were performed in nitrogen atmosphere in the temperature range $173 \leq T \leq 473 \mathrm{~K}$.

TABLE

Thicknesses of individual layer in $\AA$, the letters (a-h) describe the mode and power of magnetron sputtering process: (a) r.f. $1500 \mathrm{~W}$; (b) d.c. $1500 \mathrm{~W}$; (c) d.c. $900 \mathrm{~W}$; (d) r.f. $2400 \mathrm{~W}$; (e) d.c. $900 \mathrm{~W}$; (f) d.c. $830 \mathrm{~W}$; (g) d.c. $1000 \mathrm{~W}$; (h) d.c. $650 \mathrm{~W}$.

\begin{tabular}{c|c|c|c|c|c|c|c|c}
\hline \hline SV & Substrate & $\mathrm{Ta}(1)$ & $\mathrm{Co}(1)$ & $\mathrm{Cu}(1)$ & $\mathrm{Co}(2)$ & $\mathrm{FeMn}$ & $\mathrm{Ta}(2)$ & $\mathrm{Cu}(2)$ \\
\hline 1 & $\mathrm{Si}$ & $52(\mathrm{a})$ & $44(\mathrm{a})$ & $22(\mathrm{~b})$ & $44(\mathrm{a})$ & $85(\mathrm{a})$ & $52(\mathrm{a})$ & $5(\mathrm{c})$ \\
2 & $\mathrm{Si}$ & $52(\mathrm{a})$ & $44(\mathrm{a})$ & $22(\mathrm{a})$ & $44(\mathrm{a})$ & $85(\mathrm{a})$ & $52(\mathrm{a})$ & $5(\mathrm{c})$ \\
3 & $\mathrm{Si}$ & $52(\mathrm{a})$ & $44(\mathrm{a})$ & $22(\mathrm{~d})$ & $44(\mathrm{a})$ & $85(\mathrm{a})$ & $52(\mathrm{a})$ & $5(\mathrm{c})$ \\
4 & glass & $50(\mathrm{e})$ & $37(\mathrm{f})$ & $20(\mathrm{~b})$ & $32(\mathrm{f})$ & $100(\mathrm{~g})$ & $50(\mathrm{e})$ & $5(\mathrm{~h})$
\end{tabular}

The $G M R_{A}(T)$ and $G M R(H)=100\left[R(H)-R_{\min }\right] / R_{\min }$ dependencies are demonstrated in Fig. 1. As can be seen from Fig. 1a, b, some SV samples differ not only in $G M R_{A}$ but also in the shape of $G M R(H)$ dependencies. The $G M R(H)$ for samples 1,2 , and 4 , in contrast to sample 3 , have a square form, i.e., typical of a weak interlayer coupling $J$. According to the model proposed by Rijks [1] the unidirectional anisotropy energy can be calculated as $E_{E B}=\left(H_{1} t_{\mathrm{Co}(1)}+\right.$ $\left.\mathrm{H}_{2} t_{\mathrm{Co}(2)}\right) \mu_{0} M_{r m C_{0}}\left(M_{\mathrm{Co}}\right.$ - saturation magnetisation of cobalt, $t_{\mathrm{Co}(1)}, t_{\mathrm{Co}(2)}$, and $H_{1}, H_{2}$ are the thicknesses and the switching fields of $\mathrm{Co}(1)$ and $\mathrm{Co}(2)$ layers). The values of $H_{1}$ and $H_{2}$ are determined from $G M R(H)$ dependencies as a mean value of the switching fields for both field directions. The $m(H)$ dependencies (not presented in this paper) correlate well with $G M R(H)$ and give the same value of $H_{1}$ and $H_{2}$.

The negligible differences in $E_{E B}$ values for SV 1, 2, and 3 (Fig. 2a) indicate that for these samples the exchange anisotropy energy cannot be responsible for the observed differences in $G M R_{A} . E_{E B}$ value for SV 4 is essentially higher than that in other SV (this difference can be explained by the presence of the thicker FeMn layer in SV 4). The coupling energy between $\mathrm{Co}(1)$ and $\mathrm{Co}(2)$ layers can also be determined from $G M R(H)$ dependencies. For SV with a weak coupling $(1,2$, and 4$)$ the interlayer coupling energy can be calculated directly from $H_{1}\left(J=-H_{1} \mu_{0} M_{\mathrm{Co}} t_{\mathrm{Co}(1)}[1]\right)$. For SVs with strong coupling (no square $G M R(H)$ dependence) the $J$ value can be derived from fitting the model described in [1] to the experimentally determined $G M R(H)$ curves. 

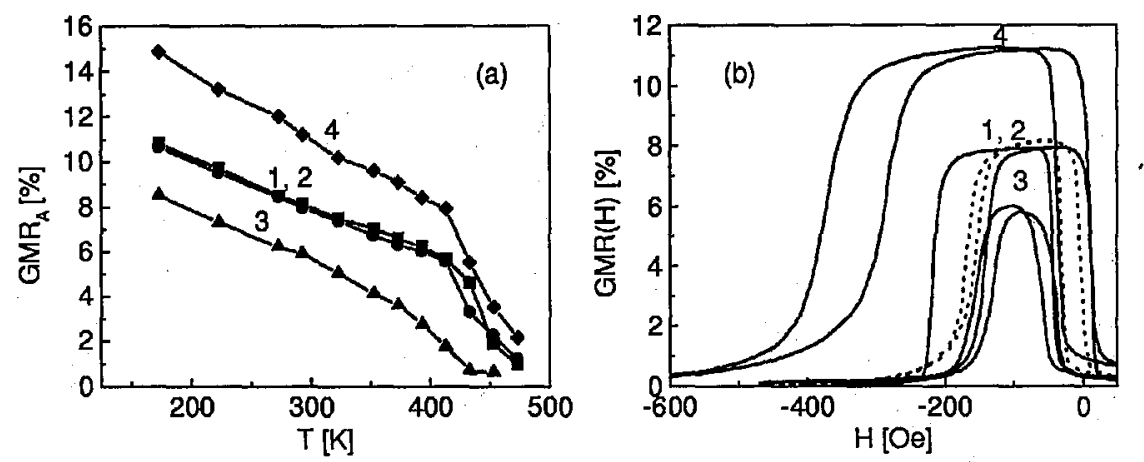

Fig. 1. Temperature dependencies of the GMR amplitude (a) for samples: 1 $(\square), 2(\bullet), 3(\Delta), 4(\diamond)$ and GMR as a function of magnetic field (b) for spin valves $1,2(\cdots), 3$, and 4 .
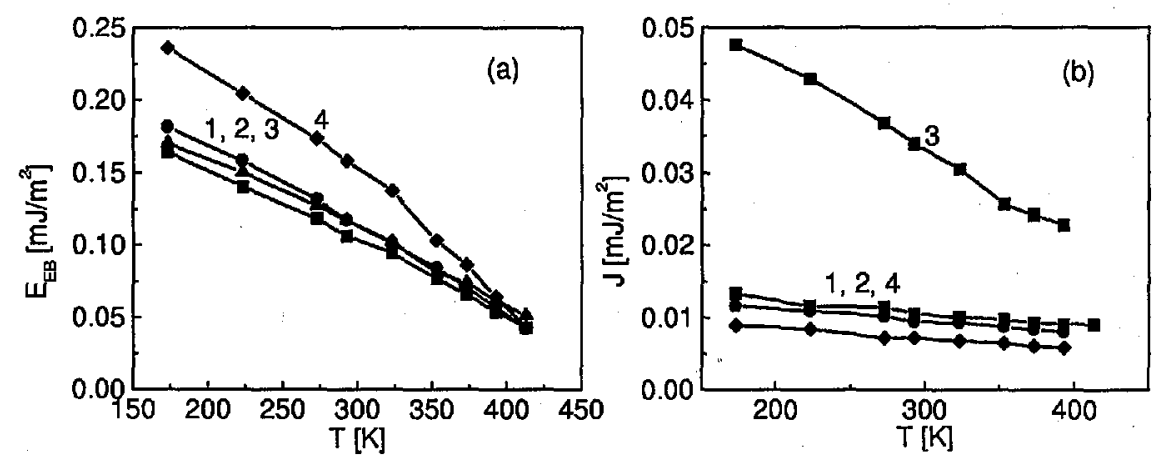

Fig. 2. Exchange anisotropy energy (a) and interlayer coupling energy (b) as a function of temperature for samples: $1(\square), 2(\bullet), 3(\triangle), 4(\diamond)$.

In Fig. $2 b$ one can see that the temperature dependencies of the interlayer coupling for SV with weak and strong couplings show essential differences in the slope. The latter results supplemented with SAXRD measurements enable us to find out the dominating source of ferromagnetic coupling (RKKY-like, magnetostatic, or caused by pinholes) in an individual SV. RKKY-like coupling can be excluded since for Cu thickness $t_{\mathrm{Cu}} \approx 2 \mathrm{~nm}$ the coupling should be antiferromagnetic $(J<0)$. The observed differences in ferromagnetic coupling for a particular $\mathrm{SV}$ cannot be explained exclusively by the existence of the magnetostatic coupling ("orange peel" coupling) since the interface roughness determined from SAXRD measurements was almost the same for all samples. Additionally the magnetostatic coupling energy $J_{M}$ for SV with Co as ferromagnetic layer should be weakly temperature dependent $\left(J_{M}(T) \propto M^{2}(T)\right.$ [3]) in the investigated temperature range. Such behaviour is observed only for samples 1, 2, and 4 (Fig. 2b). A high value of $J$ and its strong temperature dependence observed for the sample 3 suggest that in this case the coupling is caused by pinholes. It should be pointed out that for coupling caused by pinholes $J$ is strongly temperature dependent especially for pinholes with small diameter [4]. The high value of $J(j \geq 0.25)$ for the sample 3 , 

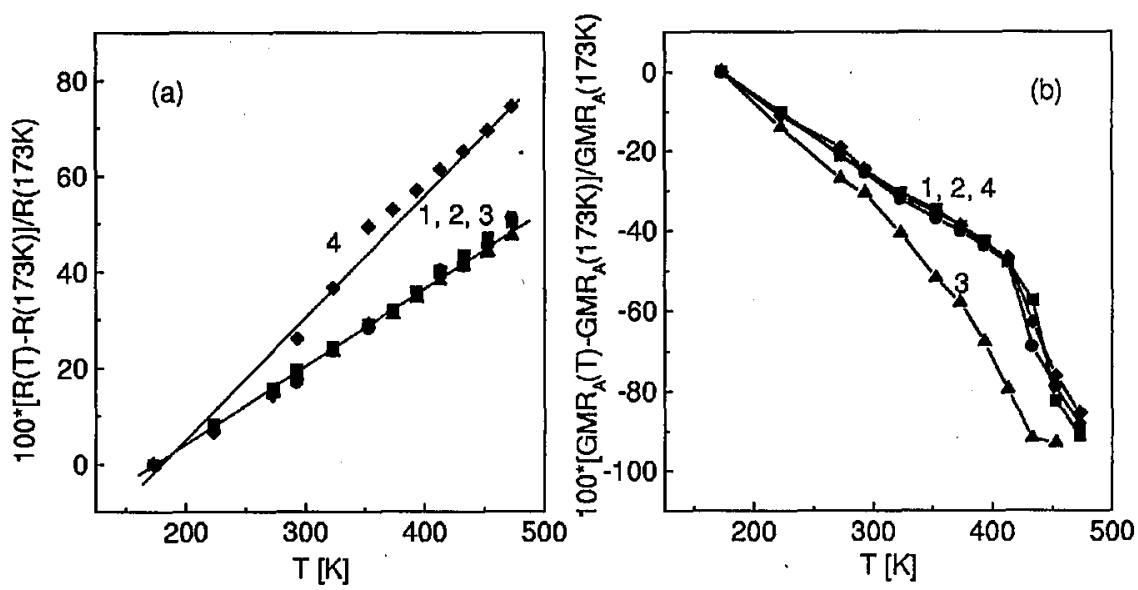

Fig. 3. Temperature changes of normalised value of resistance (a) and magnetoresistance (b), for samples: $1(\square), 2(\bullet), 3(\Delta), 4(\diamond)$.

according to the model proposed in [1], is responsible for small $G M R_{A}$ value. However, the origin of higher $G M R_{A}$ value of SV 4, in comparison with the other ones, must be different. For SV 1, 2, and $4, j \leq 0.25$, and thus $G M R_{A}$ is independent of the interlayer coupling. We suppose that in this case the most important are the differences in residual resistance and/or mean free path of electrons. Substantially stronger temperature changes of resistance observed for SV 4 in comparison to the other samples (Fig. 3a) seem to confirm such interpretation.

Finally we demonstrate in Fig. $3 \mathrm{~b}$ a comparison of the relative changes of $G M R_{A}$ as a function of temperature for SV with weak and strong coupling. As it can be seen, the temperature changes of $G M R_{A}$ are linear only for samples with $G M R_{A}$ independent of coupling strengths, i.e. for $j \leq 0.25$. This behaviour is not fulfilled for the sample 3 in whole temperature range and for the samples No. $1,2,4$ for relatively high temperatures $(T \geq 420 \mathrm{~K})$, i.e., in the vicinity of the Néel temperature of FeMn.

\section{Conclusions}

We have demonstrated that simple temperature measurements of resistance and magnetoresistance can be a useful method to verify the contribution of the ferromagnetic interlayer coupling to GMR amplitude. On the base of $G M R(H, T)$ measurements the temperature changes of the interlayer coupling can be determined and the source of the coupling can be found.

\section{References}

[1] G.S.M. Rijks, R. Coehoorn, J.T.F. Daemen, W.J.M. de Jonge, J. Appl. Phys. 76, 1092 (1994).

[2] J.L. Leal, M.H. Kryder, IEEE Trans. Magn. 32, 4642 (1996).

[3] L. Néel, Comptes Rendus 255, 1676 (1962).

[4] D.B. Fulghum, R.B. Camley, Phys. Rev. B 32, 4642 (1996). 